\title{
Novel Approach of Optimization for Economic Load Dispatch by Hybrid Particle Swarm Optimization and Genetic Approach
}

\author{
Malvika Katoch ${ }^{1}$, Harmeet Singh Gill ${ }^{2}$ \\ Student, GNDEC Ludhiana \\ Assistant Professor, GNDEC Ludhiana
}

\begin{abstract}
Hybrid Optimization using PSO (Particle Swarm Optimization) and GA (Genetic Approach) is the most significant optimization technique which has been used so far for load forecasting amongst Combined Heat and Power (CHP) generators in the power system. This Hybrid Optimization technique is designed in such a way that once we are able to forecast the load, the total fuel cost is reduced as the tendency of this optimization technique is to attain convergence. As compared to the application of using only PSO technique or only GA technique the hybrid approach of both the techniques is much more effective as well as fruitful. In this study the data of six CHP generators has been used for getting the optimum solution. The data from these generators is collated and observed using only Particle Swarm Optimization and the solution was converged as we were able to reach the optimum point. Also the same data was observed after the application of using only Genetic Approach and the solution again got converged although the convergence obtained after using only PSO and GA was not that precise as well as more time consuming. However when we used the hybrid of Particle Swarm Optimization and Genetic Approach the convergence of the solution was far better as compared to only using PSO and GA technique.
\end{abstract}

Keywords: Hybrid Optimization technique, PSO (Particle Swarm Optimization), GA (Genetic Approach), Economic Load Dispatch (ELD), Economic Dispatch

\section{INTRODUCTION}

As we know that Combined Heat and Power (CHP) unit is also known as cogeneration or distributed generation units. This technique of CHP has played a quiet an important role in the industry of utility. CHP units are used nowadays for providing electrical as well as heat energy to the consumers. The power generation efficiency generated from the conventional systems is in between 50\% and 60\%, however with the use of CHP system the efficiency has been increased by approximately $90 \%$. Not only these units have the ability to provide us higher efficiency, but they have also reduced the gaseous emission of pollutants such as $\left(\mathrm{SO}_{2}, \mathrm{CO}\right)$ and this reduction has been done by approximately 13-18\%. Combined Heat and Power Economic Dispatch (CHPED) problem is used here so that the integrated CHP system is economically available to the customers. Economic Dispatch (ED) is one of the most important optimization problems which can be used in power system operation.

$$
\begin{array}{lc}
\text { Traditional System } & \text { CHP System } \\
\mathbf{4 5 \%} \text { Efficiency } & \mathbf{7 5} \% \text { Efficiency }
\end{array}
$$

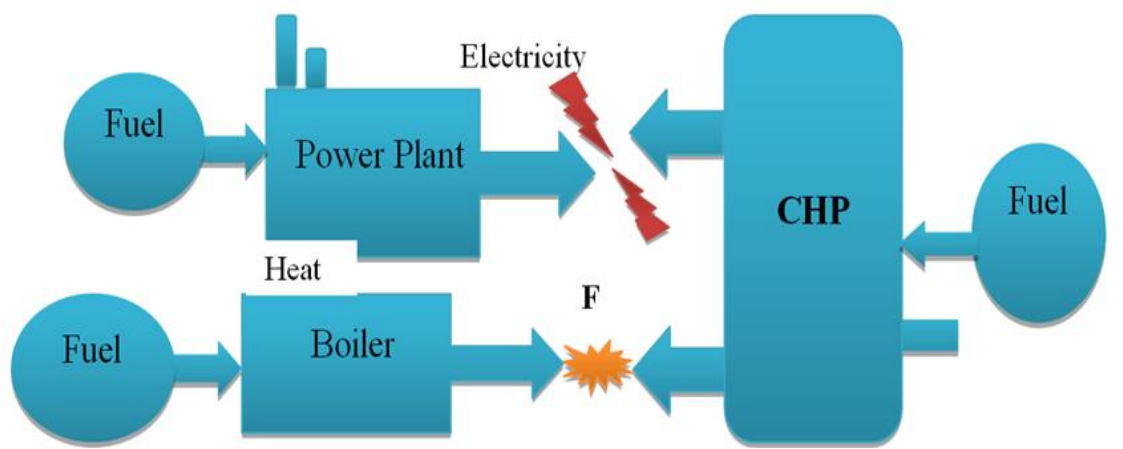

Figure 1.1 Combined Heat and Power System 


\title{
International Advanced Research Journal in Science, Engineering and Technology
}

\author{
Vol. 5, Issue 9, September 2018
}

\subsection{Economic Load Dispatch}

The term Economic Load Dispatch (ELD) is defined as the determination of the optimal solution. Optimal result means the solution is able to meet the system parameters and is converging as well. The solution is obtained from a number of electricity generation facilities and this ELD technique is important because we need it to meet the load requirements of the system with the lowest possible cost. Here we are also going to consider all the transmission as well as operational constraints. The problem of Economic Load Dispatch (ELD) can be solved with the help of specially designed computer software as this software is to be used only for this purpose. This software also considers the operational as well as the system constraints. The Economic Load Dispatch (ELD) is a very important parameter in the power system. The main aim of ELD is that it needs to minimize the total production cost of the units which are considered in a system. Economic operation strategy also helps us in the maintenance of power dispatch.

\subsection{Particle Swarm Optimization}

Particle Swarm Optimization (PSO) is a computational method (that means this method can be applied using computer software) and it optimizes a given problem by using a number of iterations. This method also helps to improve a candidate's solution to a certain measure of quality. We can also solve the problems by using a very big population of the candidate. In this population we move the particles around in the search-space with the help of a simple mathematical formulae which is designed especially for this purpose, over the particle's position and velocity. The movement of every particle is influenced by the position of its local best known position, however the movement of these particles is also guided towards its best known positions in the search-space and that particular position is updated in the software or search space as its better position as it is found by other particles. This above mentioned step helps in the movement of the swarm towards its best possible solution. PSO is basically a higher level algorithm and this algorithm makes few or no assumptions about the problem which is given to us for the purpose of optimization. PSO can search very large spaces of the candidate's solution.

\subsection{Genetic Algorithm}

Genetic Algorithm (GA) belongs to the larger class of Evolutionary Algorithms (EA) and the class of GA generates the solutions of the optimization problems by using such techniques which are inspired by the natural evolution such as inheritance, mutation, selection and crossover. These four techniques form the basis of Genetic Algorithm. The results which are obtained from Genetic Algorithm are better as compared to the conventional AI in the sense that it is quiet robust in nature. AI systems works in a better way if the inputs applied change a little bit, or they work better in the presence of reasonable noise to search a large space, multi-modal or $\mathrm{n}$ dimensional state space surface.

\section{LITERATURE REVIEW}

Haghrah, et al. (2017) - This paper involves the short-term hydro-thermal scheduling which involves scheduling of hydro and thermal generations. It helps us to minimise the fuel cost of the thermal plants while also meeting various hydraulic along with electrical constraints of the system. The problem is considered as a non-linear and non-convex problem. This problem also includes valve-point effects along with the transmission losses along with a set of equality and inequality constraints of the system. The proposed Real Coded GA approach with Random Transfer Vector-Based Mutation (RCGA-RTVM) has been applied to the two test systems having different characteristics.

Ahamad Tajudin Khader, et al. (2016) - In this paper, an algorithm is used which is a biologically-inspired method and is named as Krill Herd (KH). This method is proposed for solving the optimization problems. The KH algorithm is inspired from the simulation which is based on the herding behaviour of Krill individuals, these individuals are found in the oceans all across the world. The minimum distance between every individual (Krill) from its food and from the highest density of the Krill herd is also considered as its prime objective function for its movement. To get us more precise modelling of the behaviour of Krill we are going to add two adaptive genetic operators to the algorithm. The proposed method by the researcher has also been verified by using various benchmark problems which are usually used in the area of optimization. Further, in this literature the $\mathrm{KH}$ algorithm is also compared with eight well-known optimization methods. The $\mathrm{KH}$ algorithm is also capable to solve a wide range of benchmark optimization problems very efficiently and it has the capability to outperform the exciting algorithms also helps its users to obtain the converged solution.

Behnam Mohammadi-Ivatloo et al. (2013) - In this paper an acceleration coefficients having time varying characteristic is used along with Particle Swarm Optimization algorithm. This Time Varying Acceleration Coefficient with PSO (TVAC-PSO) has been implemented for solving Combined Heat and Power Economic Dispatch (CHPED) problem. The CHPED problem is a very complex, non-convex and non-linear optimization problem which is not at all easy to solve. The acceleration coefficients which are used in PSO algorithm varies quiet adaptively during its iterations in order to improve the solution quality obtained from the original PSO technique and it also avoids premature convergence of the solution. 


\title{
International Advanced Research Journal in Science, Engineering and Technology
}

\author{
Vol. 5, Issue 9, September 2018
}

Chao-Lung Chiang, et al. (2012) - In this paper the author has presented a technique which is called as Hybrid Differential Evolution with Multiplier Updating technique (HDE-MU) and this method is used to solve very complex Combined Heat and Power Economic Dispatch (CHPED) problems. Here transmission losses and valve-point effects of the conventional thermal generators which are used in the system under consideration are also taken into account. As compared to the past techniques including transmission losses gives this work an edge over other methods. The Hybrid Differential Evolution (HDE) has the ability to search quiet efficiently as well as actively and they explore the solutions of CHPED problem. Multiplier Updating (MU) technique is also introduced so that we can avoid the deformation of the Augmented Lagrange Function (ALF), this function has been adopted to manage constraints of the system of CHPED problem.

Anupam Masih, et al. (2018) - This paper has introduced an intellectual as well as conceptual avoidance scheme to the Particle Swarm Optimization (PSO) algorithm. It is basically a cognitive avoidance and attraction technique. The random movement of the individual particles in the given population are influenced by the personal best solution and the global best solution. These solutions might encourage the algorithm to take on an unfruitful move. This may create a delay in the convergence towards getting an optimal solution. With the similar notion as particles own their own best known position and they are attracted towards it which is called as cognitive attraction, the particles may avoid themselves from taking any move around its own known worst position and this process is called as cognitive avoidance. This above mentioned concept of cognitive attraction and avoidance is added to the standard Particle Swarm Optimization algorithm in the form of cognitive avoidance component and also with the addition of coefficient.

\section{METHODOLOGY}

The methodology used in this thesis work is PSO-GA Hybrid Optimization technique. The first step is to initialize load/power to the generators. Using GA alone and PSO alone first of all initially we will find out the total cost of fuel in the generators by applying the constraints. After that we will apply PSO-GA to the same generators and we can compare the results with PSO alone and GA alone. We can conclude after comparison that the results obtained from PSO-GA are much better and helps us to converge the solution. In this Hybrid Optimization first of all we will use apply PSO algorithm and if we are able to get the optimal solution and the solution converges then we will stop the program and if the solution does not converge then we will apply GA algorithm. After applying GA we will again check the convergence of the solution and the process continuous until the optimum solution is not found.

\subsection{Materials And Method}

SARGA (Self Adaptive Real-Coded Genetic Algorithm) is implemented to solve the CHPED (Combined Heat and Power Economic Dispatch) problem. Ant Colony Search Algorithm (ACSA) technique is also used by various researchers to solve Combined Heat and Power Economic Load Dispatch Problem. ACSA has used a positive feedback system as well as distributed computation system. ABC algorithm is very good at investigation, however it is very poor at its operation and the convergence speed offered by this algorithm is also slow in some of the cases. The above mentioned techniques have already been used and implemented earlier by different researchers to get an optimized solution. To overcome these above mentioned deficiencies, we are going to use Genetic Approach algorithm. PSO technique is usually used to solve a variety of problems of power system. PSO is used to find out the global as well as global best. Here Genetic algorithm (GA) is used for the optimization of generating units. The Genetic Algorithm is used as a base level search which is used to direct the search towards the optimum region as well as the local best position. From the mathematical results obtained, it was observed that the PSO-GA algorithm is able to provide us a better solution which leads to convergence with less computational time as well as cost. Using hybrid technique of PSO-GA we were able to find better results as compared to using only GA and only PSO technique. The computation during this Hybrid Optimization technique is also reduced.

\section{PRESENT WORK}

We are using in this present work of thesis the hybrid combination of Genetic Algorithm (GA) along with Particle Swarm Optimization (PSO) algorithm for obtaining better optimization results. Genetic Algorithm is a computational algorithm which obtains its results after running iterations. This algorithm is based on the nature of genes and their operation. Genetic Algorithm (GA) is based on the process of selection, cross-over and mutation operation as these three are its operators using which this algorithm works and using these operators we can get the optimal result. This optimization technique is based on the fitness value of genes. This algorithm is designed to support the local optimization technique which is not ever enough to produce effective solutions. To overcome this problem Hybrid Approach which is the combination of GA and PSO is proposed in this present work. The Particle Swarm Optimization (PSO) algorithm is also a meta-heuristic algorithm and it is based on the natural behaviour of swarms. This PSO algorithm is used to solve any kind of complex problem, non-linear problem so that we are able to get optimal results. 


\section{International Advanced Research Journal in Science, Engineering and Technology}

Vol. 5, Issue 9, September 2018

PSO supports the feature of Global Optimization and it helps us in providing the solution of the problem which is globally best in nature. In this present work PSO and GA work parallel hand in hand for getting us better as well as optimal solutions. Both the algorithms have different features of optimization.

\subsection{Hybrid Optimization (PSO-GA Optimization)}

As we know that GA is a discontinuous technique which is also suitable in case of the combinational problems however PSO is a continuous technique which is very poorly suited if it is to be used in case of the combination of problems. Both PSO and GA techniques can be considered as if they are going to generate new solutions in its neighbouring two parents by using crossover operator in GA and also with the help of attractions of two p (personal) best positions in PSO. PSO technique basically has two populations in it and they are known as p bests and current positions. These positions are going to allow a much greater diversity and they will probe over a single population. All Genetic Algorithms need some or other form of rearrangement, as this will allow GA to produce new solutions and those new solutions will have the legacy of their parent's success forward. As we restrict the reproduction of weak off springs (solutions), the algorithm of Genetic technique eliminates the fact that the solution and all of its successors will be eliminated. This fact will make the algorithm to converge towards a very high quality solution within very few generations. The hybrid technique (that is the combination of both these techniques which are GA and PSO), takes out the best quality of both these techniques amongst them.

\subsection{PSO-GA Algorithm}

Step 1: Initialize the total Load/Power.

Step 2: Initialize the Load/Power of the generator.

Step 3: Allocate the designated generators and calculate the total cost.

Step 4: Apply the PSO algorithm for optimization purpose.

Step 5: If the output of PSO is optimized then check the convergence else use GA and start its working with the following steps.

(a) Initialize the chromosomes.

(b) Cross over between chromosomes.

(c) Apply Roulette Selection.

(d) Check Optimization of the solution. If the solution optimizes then go to convergence otherwise the loop is still running until the objective function is not obtained.

Step 6: Check the convergence of the solution. If it converges then check the features of cost otherwise again initialize the particles and Repeat step number 5.

Step 7: If the cost is less than $\Delta \mathrm{C}$ then stop.

\section{RESULTS}

In this section the results which are obtained after applying only PSO algorithm, only GA algorithm and the hybrid combination of PSO-GA algorithm is shown. Also the comparison of the results is done with the above mentioned algorithms is presented in this section. This result is calculated on the heat only units and power only units on GA, PSO and GA with PSO. As we are considering CHP system here so there is a dual dependency of heat as well as power production in CHP units and this makes the problem more complicated, hence to solve this problem we are using the Hybrid Optimization Technique by combining PSO and GA. In CHP systems the power production limits of the units are dependent to the unit heat production and vice-versa is also true. Here the power only unit is the one which acts as a normal generator and uses mechanical energy to produce electricity and produces heat energy as its waste product and this waste heat is used as an input in the heat only units. Here Total Heat is in Wth, total power is in W and total cost is in ₹.

Table 5.1 Generator comparison values

\begin{tabular}{|c|c|c|c|c|c|c|}
\hline Approaches & $\begin{array}{c}\text { Generator 1 } \\
\text { (MW) }\end{array}$ & $\begin{array}{c}\text { Generator 2 } \\
\text { (MW) }\end{array}$ & $\begin{array}{c}\text { Generator 3 } \\
\text { (MW) }\end{array}$ & $\begin{array}{c}\text { Generator 4 } \\
\text { (MWth) }\end{array}$ & $\begin{array}{c}\text { Generator 5 } \\
\text { (MWth) }\end{array}$ & $\begin{array}{c}\text { Generator 6 } \\
\text { (MWth) }\end{array}$ \\
\hline GA & 75.332 & 79.7093 & 49.11 & 0.0321 & 331.56 & 14.758 \\
\hline PSO & 75.332 & 79.7093 & 49.118 & 0.205 & 69.0432 & 125.48 \\
\hline GA with PSO & 75.332 & 79.7093 & 49.11 & 0.0253 & 778.27 & 0.0169 \\
\hline
\end{tabular}




\section{International Advanced Research Journal in Science, Engineering and Technology}

Vol. 5, Issue 9, September 2018

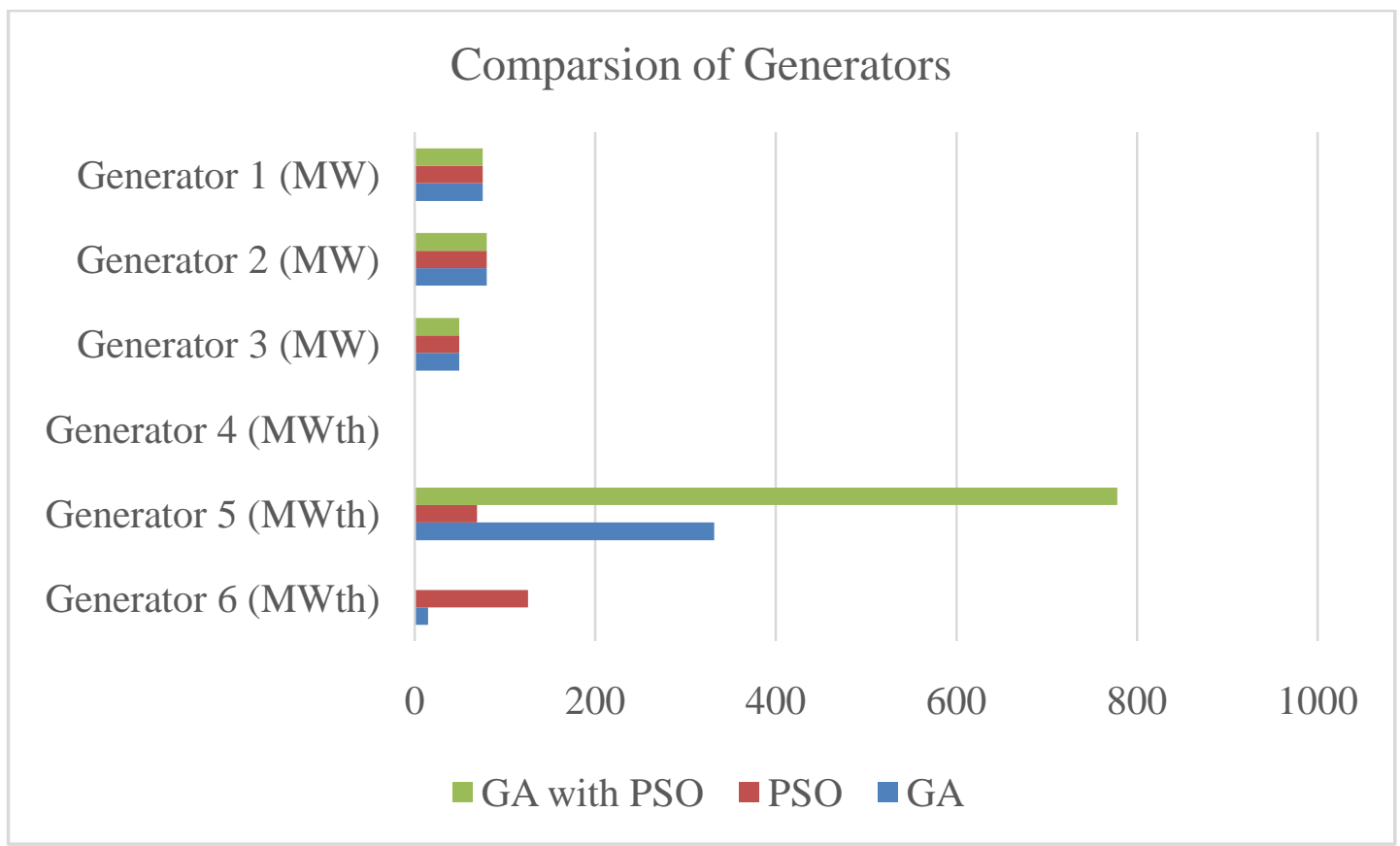

Figure 5.1 Generators Comparison

This above mentioned graph shows us the comparison between all six generators after the application of only PSO, only GA and GA-PSO hybrid techniques. After comparison these results clearly indicate that the hybrid PSO-GA algorithm has the ability to provide us much better solution as compared to only PSO and only GA algorithms.

Table 5.2 Cost Results Using Different Algorithms

\begin{tabular}{|c|c|c|c|}
\hline Approaches & $\begin{array}{c}\text { Heat Production } \\
\text { Cost }(₹)\end{array}$ & $\begin{array}{c}\text { Power Production } \\
\text { Cost }(₹)\end{array}$ & Total cost(₹) \\
\hline GA & 725.09 & 1002.59 & 1727.68 \\
\hline PSO & 625.03 & 472.74 & 1024.67 \\
\hline GA with PSO & 515.2 & 320.13 & 835.33 \\
\hline
\end{tabular}

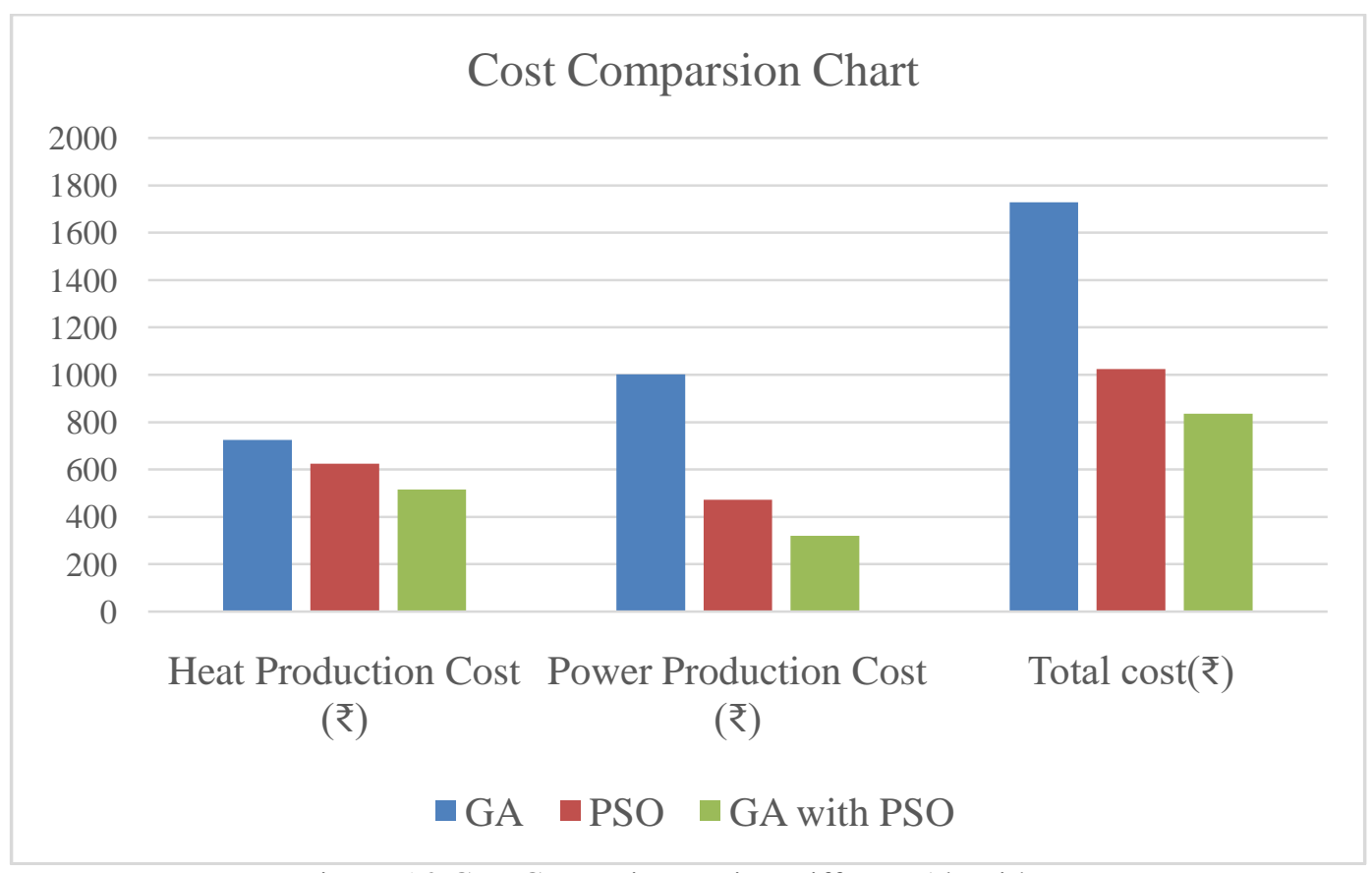

Figure 5.2 Cost Comparison Using Different Algorithms 


\section{International Advanced Research Journal in Science, Engineering and Technology}

Vol. 5, Issue 9, September 2018

It can be clearly observed that the total cost of the system is much less after implementing PSO with GA as compared to only PSO and only GA technique. This means that the hybrid approach towards this system is a much better approach.

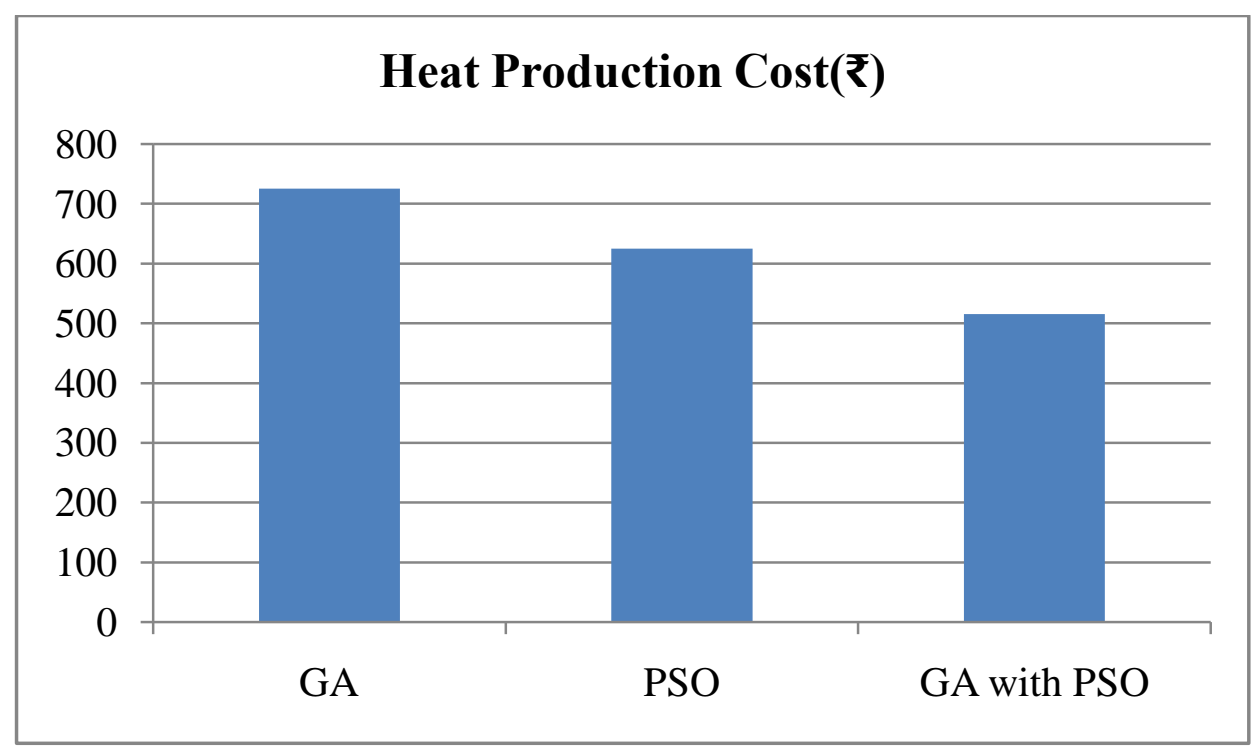

Figure 5.3 Heat Production Cost after Implementing Different Algorithms

The figure 5.3 depicts the cost of heat in the different algorithms. In this graph $\mathrm{x}$-axis represents the algorithmic approach and y-axis shows the value of cost.

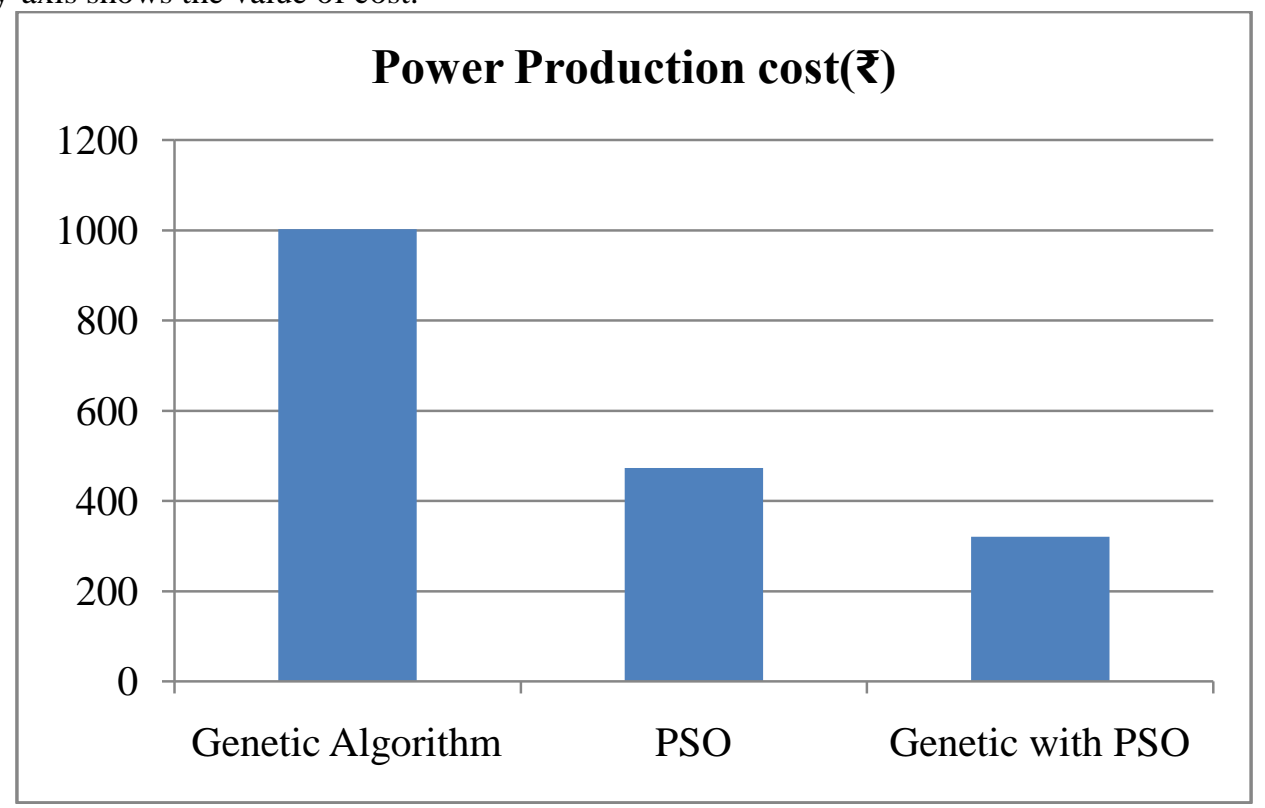

Figure 5.4 Power Production Cost after Implementing Different Algorithms

In figure 5.4 the $\mathrm{x}$-axis shows the approach used in the work and their comparison and $y$-axis represents the cost of the algorithm. 


\section{International Advanced Research Journal in Science, Engineering and Technology}

Vol. 5, Issue 9, September 2018

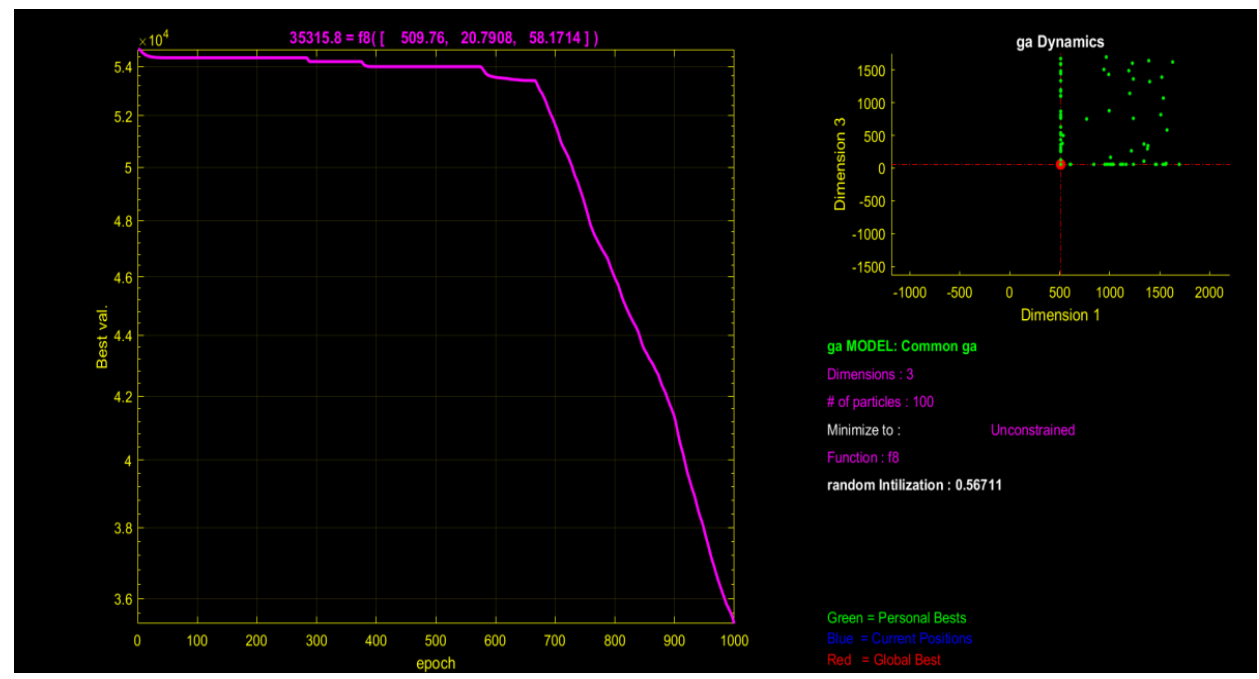

Figure 5.5 MATLAB Result after Running GA

We ran GA on a given population and the results obtained after running the Genetic algorithm in MATLAB is shown above. The obtained result is dynamic in nature and the result shown above is one of the results we have obtained after running GA.

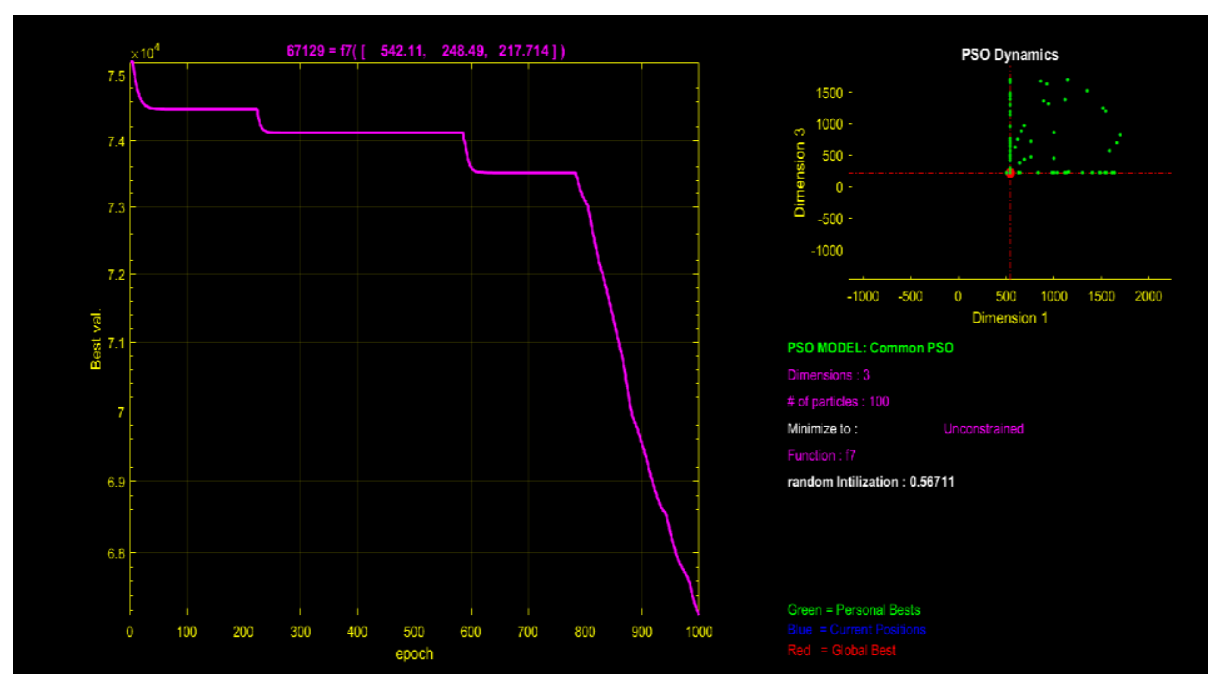

Figure 5.6 MATLAB Result after Running PSO Algorithm

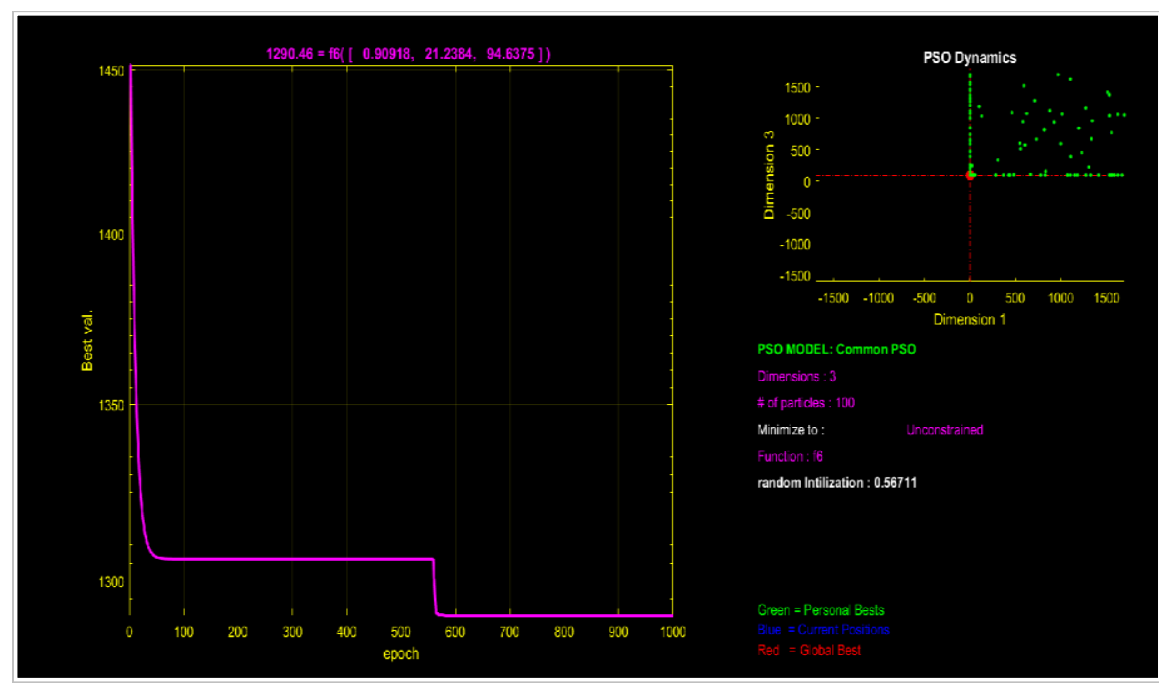

Figure 5.7 MATLAB Result after Running PSO-GA Hybrid Optimization 
Vol. 5, Issue 9, September 2018

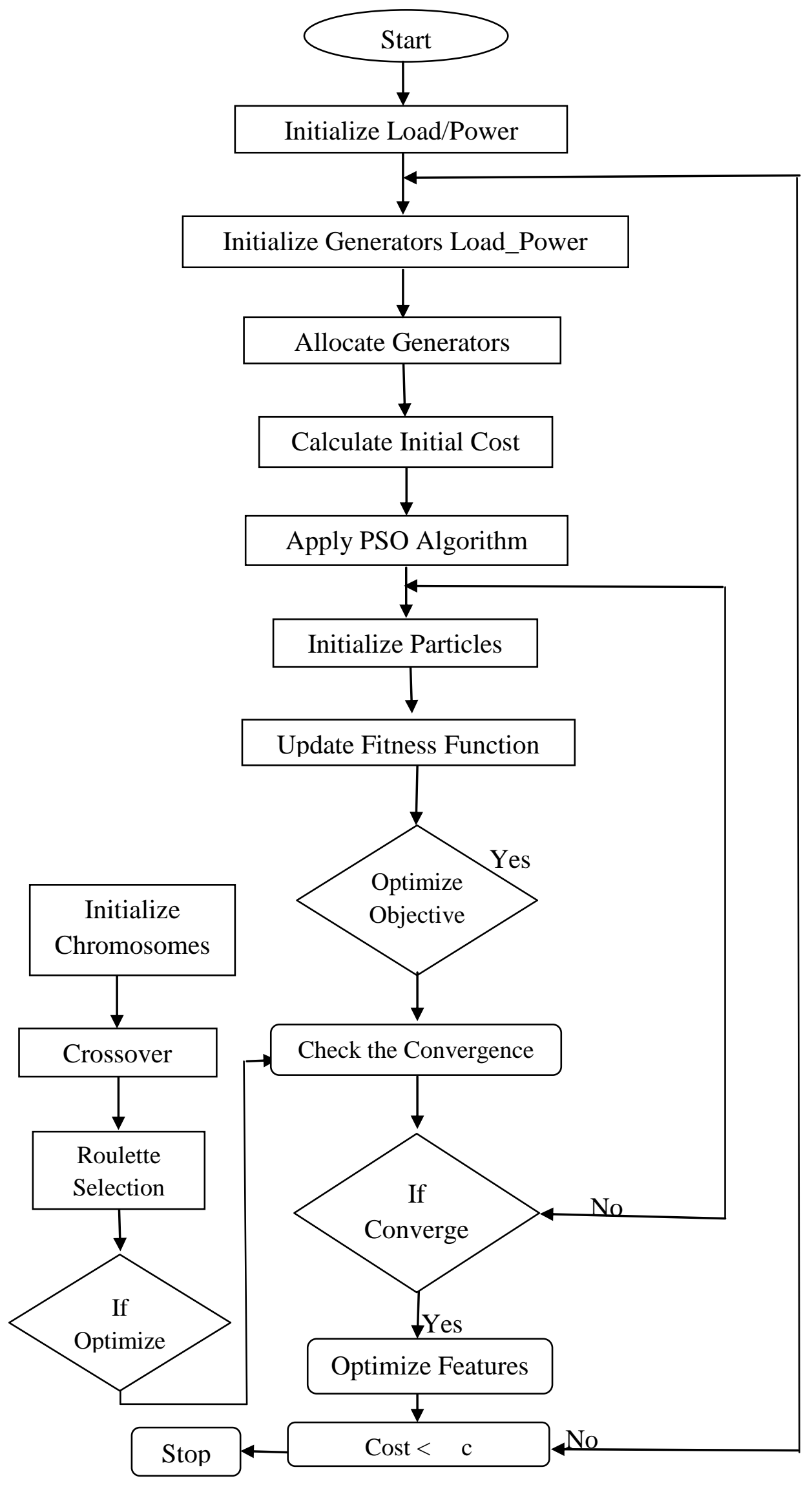




\title{
International Advanced Research Journal in Science, Engineering and Technology
}

\author{
Vol. 5, Issue 9, September 2018
}

\section{CONCLUSION}

It is almost impossible to have such an algorithm which is suitable in all kind of situations every time. Therefore it is very important to consider the situations for which the further study is required. There are few areas which are suggested in this section which can be further explored so that the total fuel cost can be reduced. In the systems where we are using the hybrid combination of algorithms, it depends on the type of application, design, and feasibility. There are many different strategies that can be adopted here. However, the structure of algorithm includes the hybrid optimization process and the practical application of this scheme might demand a higher computational integrity. Hybrid algorithms can provide us good results with the combinations of various practical applications with the help of these energy sources. There is a great scope for us to implement the suggested hybrid power system configurations in the practical applications. The technology which is used for the hybrid optimization is changing rapidly with time and with the changing demand of energy, also the size and scope of problems that can be solved also increases steadily. The complexity of technology also varies day by day. There are many techniques which are available in the market but we need to see that by looking into the fact that which combination of techniques is beneficial for us and how can we improve the results there by decreasing the total computational time we well as total fuel cost. We foresee a huge demand for simplification purpose with the use of combinational optimization technology. The increasing power demand is forcing us to improve our ability to provide complex hybrid solutions. These demands place emphasis on the universal modelling languages which are flexible in nature and can withstand non-convex, non-linear and other higher level complex problems. Nowadays optimization technique is being used in several fields which are related to our day to day life. The use of hybrid optimization is not only restricted to a one specific area. In fact it can be used in power systems, traffic signals, agriculture, medicine etc. With wider audience optimization and especially hybrid optimization is a hot topic. In electrical engineering the problem becomes more complex because of the introduction of losses, valve point effects etc. and this also impacts the fuel consumption. Therefore with the use of hybrid optimization technique not only we have the option to reduce the fuel cost but we can also save up our time of computation along with the total cost reduction. If we compare the above MATLAB graphs then we can clearly see that the convergence is obtained quiet accurately after running the Hybrid Optimization of PSO-GA.

\section{REFRENCES}

[1]. Ahamad Tjudin Khader, Mohammad Abualgiah, Mohammed A. Awadallah and Mohammed Azmi Al-Betal (2016) “A Comprehensive Review: Krill Herd Algorithm (KH) and its applications", Springer Innovations in Communication and Computing, vol.44, pp. 437-446.

[2]. Amir Mahyar Khorasani, Majid Ebrahim Warkiani and Mohsen Asadnia (2017) “ An Accurate PSO-GA Based Neural Network to Model Growth of Carbon Nanotubes", Journals of nano materials, vol.2017, pp.6.

[3]. A.K Al- Othman, J.K Sykulski and J.S Al-Sumait (2007), “Application of Pattern Search Method to Power System Valve-point Economic Load Dispatch", International Journal of Electrical Power and Energy Systems, vol.29, pp. 720-730.

[4]. Anupam Masih, Apurva Gautam and A. Ashok (2018), “ Implementation of Smooth and Non- smooth Fuel Cost Function for ELD Using Cuckoo Search Method", International Journal of Current Engineering and Technology, vol.8, pp.3.

[5]. Anupam Masih, A. Ashok, Prinu C. Phillip and Sudhanshu Tripathi (2017), “Performance Analysis of Various Soft Computational Techniques for Economic Load Dispatch”, Asian Journal of Convergence in Technology, vol.2, pp. 1146-2350.

[6]. Abbas Rabiee, Behnam Mohammadi Ivatloo and Mohammad Moradi-Dalvand (2013), "Combined Heat and Power Economic Dispatch Problem Solution Using Particle Swarm Optimization with Time Varying Acceleration Coefficients”, Electric Power System Research, vol.95, pp.9-18.

[7]. A. Carrascal, E. Perea, I Cobelo and Nerea Rviz (2015), "A Novel Optimization Algorithm for Efficient Economic Dispatch of Combined Heat and Power Devices", International Council for Research and Innovation in Building and Construction, vol.111, pp. 507-514.

[8]. Hamdi Abdi, M. La Scala and Soheil Derafshi (2017), "Optimal Operation of Multicarrier Energy Systems Using Time Varying Acceleration Coefficient Gravitational Search Algorithm", vol.138, pp. 861-872.

[9]. A. Hardiansyah (2013) “Artificial Bee Colony Algorithm for Economic Load Dispatch Problem", IAES International Journal of Artificial Intelligence, vol. 2, pp. 90-99.

[10]. Chao Lung Chiang (2012), "Hybrid Differential Evolution Based Multi-objective Approach for Hydrothermal Power Systems", Applied Mechanics and Materials, vol.212-213, pp. 1009-1014.

[11]. Anastasios G. Bakitzis, Ioannis G. Damousis and Petross Dokopouloas (2003), "Network Constrained Economic Dispatch Using Real Coded Genetic Algorithm", vol.18, pp. 198-205.

[12]. A.M Elaiw, A.M Shehata and X. Xia (2013), “Combined Heat and Power Dynamic Economic Dispatch with Emission Limitations Using Hybrid DE-SQP Method", Abstract and Applied Analysis, vol. 2013, pp. 10.

[13]. Jin-Hua She, Yong He and Zhi-Yong (2012), "Hळ Static Output Feedback System”, Asian Journal of Control, vol.14, pp. 1505-1513.

[14]. A. Haghrah, Behnam Mohammadi Ivatloo and M Nazari Heris (2017), "Optimal Short-term Generation Scheduling of Hydrothermal Systems by Implementation of Real Coded Genetic Algorithm Based on Improved Mutation”, Institution of Engineering and Technology, vol. 128 , pp. 77-85.

[15]. Haiyan Lu, Pichet Sriyanyong, Tharam Dillon and Yong Hua Song (2010) "Experimental Study of a New Hybrid PSO with Mutation for Economic Dispatch with Non-smooth Cost Function", International Journal of Electrical Power \& Energy Systems, vol.32.9, pp.921-935.

[16]. M. Basu (2013) “Artificial Bee Colony Optimization for Multi-area Economic Dispatch”, International Journal of Electrical Power \& Energy Systems, vol.49, pp. 181-187.

[17]. Edward Williams and Mohammed Arezki (2015), "Cuckoo Optimization Algorithm with Penalty Function for CHPED Problem", Handbook of Neural Computation, vol.93, pp. 1711-1718.

[18]. Jagdev Singh, Sanjay Prakash Sood, Vijay Kumar, Yaduvir Singh (2016) "Performance Analysis and Comparison of Optimal Economic Load Dispatch Using Soft Computing Techniques", International Journal of Advanced Computer Science and Application, vol.7, pp.8.

[19]. M.V Rao and Suman M (2016) "Optimal Dispatch of Power Generation Using Artificial Neural Network System", vol. 8, pp.1939-1943.

[20]. Ibrahim M Diaa, Mahmoud A Altva, Niveen M Badra (2017) “Classical and Artificial Optimization Techniques to Enhance Power System Operation", International Journal of Engineering Applied Sciences and Technology, vol.2, pp.221-226. 